von den Ambitionen Teslas mindestens eine weitere Fabrik für prismatische Zellen in Japan und neue Standorte jeweils in den starken ElektromobilitätsLändern. Das japanische Unternehmen schreibt derzeit rote Zahlen und die Tesla-Offensive könnte Panasonic gelegen kommen.

\section{VIRTUELLE PREISE}

Zur Momentaufnahme zählt auch, dass Volkswagen nach Aussagen eines hochrangigen Entscheiders im Konzern, trotz eindeutig positiver Statements [6] die Zusammenarbeit mit Panasonic derzeit auf den Prüfstand stellt. VW wie alle OEMs sagen, dass die prismatischen Zellen einfach noch zu teuer sind. Zudem steht die hochgelobt Zelle von Panasonic [6] auch unter technischen Gesichtspunkten in der Kritik. Sie neigt zum Aufblähen und muss deswegen im Stack mit hohem Druck verspannt werden. Das werden die Batteriezellentwickler in Wolfsburg im Zuge der Entwicklung und der Freigabe allerdings eingeplant haben.

Die Strategie der Wolfsburger ist durchschaubar: Technisch muss man erst einmal in einem sicheren Hafen landen, koste es, was es will. Preisverhandlungen kommen dann später. Doch kann VW in Anbetracht des ausgedünnten Anbietermarkts überhaupt pokern? Der Konzern hatte Samsung bereits auf dem Plan, für den Aufbau einer „Second Source“. Das koreanische Unternehmen ist technologisch gerüstet und will Marktanteile erobern. Es ist anzunehmen, dass dies im Rahmen von Preiszugeständnissen gelingen kann. Von einem realen Preisgefüge in der Batteriebranche kann man demzufolge nicht sprechen. Die Preise sind virtuelle Wunschpreise, das hört man aus den Kreisen der Batteriehersteller.

An dieser Stelle ist zu bemerken, dass bei allen erwähnten Preisdiskussionen eine entscheidende Bewertung auf der Strecke bleibt: realistische, und damit kostendenkende Batteriezell-Preise. Hier wird gnadenlos runtergerechnet, sagt ein Insider. Für die künftige Innovationsfähigkeit solcher Akkuzellhersteller kann sich dies sehr negativ auswirken. Denn die Investitionen in die nächste Batteriegeneration sind enorm.

\title{
„NICHT AUF TEUFEL KOMM RAUS RUNTERRECHNEN“
}

\section{Vor wenigen Jahren wurde die kWH eines Lithium-Ionen-Akkus mit 800 bis 1000 Euro veranschlagt. Dass die mit 200 Euro/kWh immer noch hohen Kosten für Lithium-Ionen- Akkus allen OEMs Kopfschmerzen bereiten, ist nachvollziehbar, das war aber auch vorhersehbar. Bei einer so sensiblen und noch jungen Technik und ersten Serienhochläufen sollte die angestrebte hohe Sicherheit Vorrang haben. Eine simple Lösung wie eine 18650-Rundzelle beeindruckt zwar, doch handelt es sich hierbei nicht eher um eine Übergangslösung?}

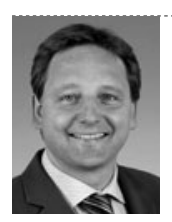

MARKUS SCHÖTTLE

Stellvertretender Chefredakteur

\section{LITERATURHINWEISE}

[1] Roland Berger Strategy Consultants veröffentlichte im April 2012 eine Studie zu Lithium-IonenAkkumulatoren

[2] http://www.springerprofessional.de/in-neuemlithium-ionen-akku-ranking-liegt-panasonic_sanyoauf-platz-1/4969160.html

[3] http://www.springerprofessional.de/gigafabrikvon-tesla-bedroht-automobile-batterieindustrie/ 4987920.html
[4] Horak, S; Pascha, W.: Im Kapitel Wettbewerbsfähigkeit der japanischen und koreanischen Automobilindustrie im Übergang zur Elektromobilität aus dem Buch „Schritte in die künftige Mobilität“. Springer Fachmedien Wiesbaden. S. 491 [5] Index Elektromobilität 2014, Roland Berger Strategy Consultants

[6] Schöttle, M.: Nicht auf die Superzelle warten. In: Interview mit Dr. Matthias Ullrich, Volkswagen, ATZelektronik 8 (2013), Nr. 3 